\title{
Temporary Tracking Specifications Conversion to Obtain the Upper Frequency Limit
}

\author{
Javier Joglar Alcubilla \\ Avionics Department, Barajas Institute, Avda. América 119, \\ 28042, Madrid, Spain \\ jjoglar@educa.madrid.org
}

\begin{abstract}
In robust control techniques where the control problem is in the frequency domain, it is required to specify process plants and disturbances in this domain, but also the limits and restrictions imposed originally in temporary form. A good technique to translate specifications from time to frequency will positively influence in the degree of overdesign and amount of iteration to achieve adequate results. The "inconvenient" is that for each type of specification is necessary to develop a specific conversion technique. The relevant technical for tracking specifications translation referred to the upper limit frequency is presented here. It will use approaches based on subcritical second order systems or an envelope approach with higher order systems.
\end{abstract}

Keywords: Time domain, Frequency domain, Quantitative feedback theory, Tracking, Overdesign, Inverse interpolation

\section{Introduction}

When transferring specifications from one domain to another is interested maximum precision and, in particular, from time to frequency, since in many control problems this conversion affects in later stages [16]. So if it is not done properly, it will generate a cumulative error, from the beginning of the problem. Parameter translation from frequency domain $(W D)$ to time domain $(T D)$ has direct application functions [3], so that, temporal verification of processed results in the $W D$ can be carried out after frequency analysis without problem, in most cases. However, contrary, the treatment of the above functions is simply not possible in reverse form, so approximation techniques, not trivial at all, should be applied or classical hypotheses for simplification of the problem, with the consequent efficiency reduction in this case [13].

Control methodologies whose design phase is developed in the $W D$, as in "Quantitative Feedback Theory" $(Q F T)[7,8,9]$, require a translation in frequency of the specifications imposed in the $T D$ as accurate as possible, since any deviation will affect to the bounds definition stage, critical when synthesize the controller [15].

Traditionally, the low accuracy contributed to the specifications conversion from $T D$ to WD by techniques such as Krishnan and Cruickshanks' [12], D'Azzo and Houpis models [4], or the Franchek and Herman's procedure [5], is supplemented with specification, design and analysis processes of manual iteration, ending when the designer understands as acceptable the results achieved.

In control methodologies whose design stage is performed in frequency [17], but requirements are from temporal domain, a rigorous technical of transfer specifications from $T D$ to $W D$ is important for the following reasons:

- The desired controller can be set to more precise conditions, which generates less iteration until a solution is achieved, providing adequate results in both frequency and time domains. 
- The "uncertainty" presented by the requirements demanded in the design phase of the controller will be minimal, so that, the degree of overdesign and thus the feedback cost are reduced [6].

However, the specifications conversion used is necessary to be a specific technical respect to the type of requirement is intended to translate. It will not be the same talking about tracking specifications, either of specifications for regulation (sensitivities); it will be necessary also to distinguish between coupling and decoupling of controlled variables for tracking and, even, if the limit to define is upper or lower. In any case, depending on the characteristics of the specification, so it should be the specific transfer process, thus a conversion is achieved with high accuracy and reliability, in every situation.

For tracking purposes, we can define the coupling of an output variable with respect to a reference input in two ways:

- From two limits, lower and upper, where the response should be between both two.

- From a single limit, upper or lower and, then, the response must take values lower or higher, respectively, from the limit considered.

Anyway, the specifications conversion for tracking between domains involves defining a specific one for every kind of limit, upper and lower, as the input parameters and output requirements are different in each case.

It is presented here an automatic transfer procedure for tracking specifications from time domain to frequency domain, specific to achieve the upper frequency limit. This limit is approximated to a $2^{\text {nd }}$ order subcritical damped transfer function $(T F)$ with characteristic parameters " $\omega_{n}$ " (natural frequency) and " $\zeta$ " (damping coefficient). To obtain rise and settlement times required is applied the necessary inverse interpolation, which will consist of a technique of successive approximations based on "Newton's Method for $5^{\text {th }}$ order Ascending and Descending Differences", described with detail in [10].

The proposed technique will work with step inputs, providing $2^{\text {nd }}$ order transient responses with subcritical damping, representing the upper frequency limit of a tracking specification. Additionally, this technique can provide manual temporary tracking specifications translation to frequency domain with higher order system solution, through an envelope approach.

\section{Technical for Tracking Specifications Translation of the Upper Frequency Limit}

The method for tracking specifications translation from $T D$ to $W D$ presented here [10], is defined by the following relationship,

$$
\begin{gathered}
T D_{\text {final }}\left(y_{\text {max }}, r_{\text {max }}, d e v\right) \\
\downarrow \\
W D\left(\text { Num }_{\text {max }}, D e n_{\text {max }}\right)
\end{gathered}
$$

The $T D$ input parameters describe, the maximum amplitude $y_{\max }$ of the step response, which tracks the step input with maximum amplitude $r_{\text {max }}$, where dev is the settlement channel of the response (admissible tolerance). $W D$ equivalent parameters determine the $T F$ of the upper limit $T_{U p p}(j \omega)$ given by,

$$
T_{U p p}(j \omega)=\frac{N u m_{\max }}{D e n_{\max }}(j \omega)
$$

The result will be characterized by a defined maximum overshoot $M p$, function of the input parameters $y_{\max }$ and $r_{\max }$, such that, 


$$
M_{P}=\frac{y_{\max }-r_{\max }}{r_{\max }}
$$

If we consider the overshoot $M p$ defined for subcrital systems as in [14], i.e.:

$$
M p=e^{-\frac{\zeta \pi}{\sqrt{1-\zeta^{2}}}}
$$

Which corresponds a minimum damping coefficient $\left(\zeta=\zeta_{\min }\right)$,

$$
\zeta_{\text {min }}=\left[\frac{(\ln M p / \pi)^{2}}{1+(\ln M p / \pi)^{2}}\right]^{1 / 2}
$$

On the other hand, for rise time $t r$ and settlement time $t s$ of the response, as what is sought is an upper frequency limit, the temporal equivalences are minimum values. However, for practical purposes must be imposed,

- Reference upper times $t r_{u p p}$ and $t s_{u p p}$ (for example, if the user does not indicate other, by default $5 \mathrm{~s}$ and $20 \mathrm{~s}$, respectively), to seek associated $\omega_{n}$ with minimum times;

- To avoid a singularity by using inverse interpolation, minimum times must be limited, which can be nulls in theory, but not so in practice.

The sequence of steps for tracking specifications translation of the upper frequency limit is as follows,

1. Determination of minimum damping coefficient $\zeta_{\min }$. From input parameters $y_{\max }$ and $r_{\max }$, the maximum overshoot $M p$ (3) is obtained, which inserted into equation (5) provides $\zeta_{\min }$.

2. Find out the relationship $\omega_{n}\left(t r, t s, \zeta_{\min }, d e v\right)$. Considering that $2^{\text {nd }}$ order systems with the same $\zeta$, but different $\omega_{n}$, are characterized by the same overshoot $M p$, so they have the same relative stability. The time constant of systems with the same relative stability is given by,

$$
T=\frac{1}{\zeta \omega_{n}}
$$

It can be expressed for generic $t r$ and $t s$, respectively, as

$$
\begin{aligned}
& \operatorname{tr}\left(\omega_{n}\right)=\frac{1}{\zeta \omega_{n}} k_{r} \\
& t s\left(\omega_{n}\right)=\frac{1}{\zeta \omega_{n}} k_{s}
\end{aligned}
$$

Parameters $k_{r}$ and $k_{s}$ depend on the tolerance band value imposed [14]. If we use the value $\omega_{n}=1$, the above equations (7) and (8) can be rewritten as,

$$
\begin{aligned}
& \operatorname{tr}\left(\omega_{n}=1\right)=\frac{1}{\zeta} k_{r} \\
& t s\left(\omega_{n}=1\right)=\frac{1}{\zeta} k_{s}
\end{aligned}
$$

Combining (7) with (9) and (8) with (10), the pair of natural frequency values $\omega_{n r}$ and $\omega_{n s}$, associated to $t_{r}$ and $t_{s}$, respectively, are obtained in the following way, 


$$
\begin{aligned}
& \frac{\operatorname{tr}\left(\omega_{n}=1\right)}{\operatorname{tr}\left(\omega_{n r}\right)}=\omega_{n r} \\
& \frac{t s\left(\omega_{n}=1\right)}{t s\left(\omega_{n s}\right)}=\omega_{n s}
\end{aligned}
$$

Note that $\omega_{n r}$ is a specific $\omega_{n}$ for $t r$ required and, so for $\omega_{n s}$ respect to $t s$.

2a. Applying equation (11) with $\operatorname{tr}$ and $\zeta_{\min }$, it is achieved,

$$
\frac{\operatorname{tr}\left(\omega_{n}=1, \zeta_{\min }\right)}{\operatorname{tr}\left(\omega_{n r}, \zeta_{\min }\right)}=\omega_{n r}\left(t r, \zeta_{\min }\right)
$$

Being $\operatorname{tr}\left(\omega_{n r}, \zeta_{\min }\right)$ an input parameter required and $\operatorname{tr}\left(\omega_{n}=1, \zeta_{\min }\right)$ calculated with a successive approximation technique, finally $\omega_{n r}\left(t r, \zeta_{\text {min }}\right)$ is obtained from equation (13).

2b. Similarly, applying equation (12) for $t s$ and $\zeta_{\min }$, it is achieved,

$$
\frac{t s\left(\omega_{n}=1, \zeta_{\min }\right)}{t s\left(\omega_{n s}, \zeta_{\min }\right)}=\omega_{n s}\left(t s, \zeta_{\min }\right)
$$

With the input parameter $t s\left(\omega_{n s}, \zeta_{\min }\right)$ and $t s\left(\omega_{n}=1, \zeta_{\min }\right)$ calculated using a successive approximation technique, $\omega_{n s}\left(t s, \zeta_{\min }\right)$ is obtained from equation (14).

The way proposed to determine $\operatorname{tr}\left(\omega_{n}=1, \zeta_{\min }\right)$ in $\left.2 \mathrm{a}\right)$ and $t s\left(\omega_{n}=1, \zeta_{\min }\right)$ in $\left.2 \mathrm{~b}\right)$ is applying "differences Newton method" [2]. For this, use as interpolation function the typical step $\operatorname{sign} f_{\text {step }}(t)$, i.e.:

$$
f_{\text {step }}(t)=1-\frac{e^{-\zeta \omega_{n} t}}{\sqrt{1-\zeta^{2}}} \operatorname{sen}\left(\omega_{n} \sqrt{1-\zeta^{2}} t+\arccos \zeta\right)
$$

For each $t r$ and $t s$, values of $f_{\text {step }}\left(t_{r}\right)$ and $f_{\text {step }}\left(t_{s}\right)$ are given. Observe that we want to obtain temporal parameters applying inverse interpolation in (15), with $\omega_{n}=1$ and $\zeta=\zeta_{\text {min }}$ so, only there is one unknown parameter in the equation each time.

3. Choose for $\omega_{n}\left(\zeta_{\min }\right)$ the biggest value between $\omega_{n r}\left(t r, \zeta_{\min }\right)$ and $\omega_{n s}\left(t s, \zeta_{\min }\right)$, being this the most restrictive condition for the temporary joint requirements $(t r, t s)$.

4. Calculate $\omega_{n}\left(\zeta_{\min }, t r, t s, d e v\right)$, varying $t r$ and $t s$, with $t r_{u p p} \geq t r \geq t r_{\min }$ and $t s_{u p p} \geq t s \geq t s_{\min }$, which will be named as $\left(\omega_{n}, \zeta_{\min }\right)$ pairs or double vector $w d\left(\omega_{n}, \zeta_{\min }\right)$.

To do this, decrease the value of $t r$ and $t s$ from its initial input upper values, $t r_{u p p}$ and $t s_{\text {upp }}$, respectively, to a practical minimum limit, for example, using the following routine:

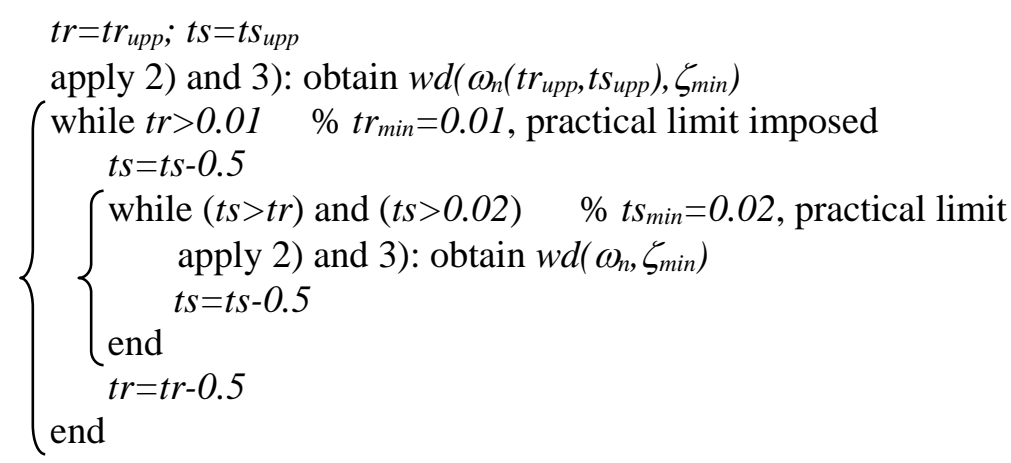

Observe that for a specific $t s$ value, if you reduce $\omega_{n}$ value respect to the one given for the associated pair $\left(\omega_{n}, \zeta_{\min }\right)$, to maintain $t s$ value, $\zeta_{\min }$ must be bigger $(M p$ decreases, 
which is valid). So, allowed frequency response is given for the area underneath the curve $\left(\omega_{n}, \zeta_{\min }\right)$. See Figure 1.

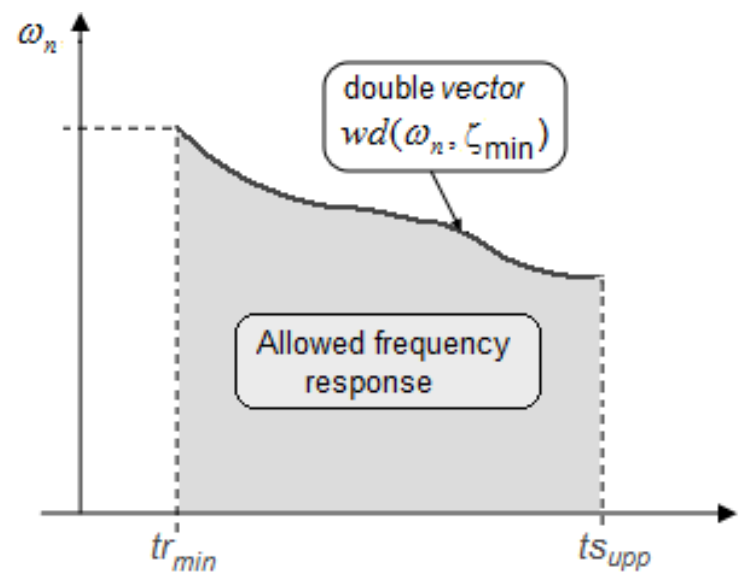

Figure 1. Graphical Representation of $w d\left(\omega_{n}, \zeta \min \right)$

5. Define $2^{\text {nd }}$ order curves $T(j \omega)$, such that,

$$
T(j \omega)=\frac{\omega_{n}^{2}}{s^{2}+2 \zeta_{\min } \omega_{n} s+\omega_{n}^{2}} \quad \text { with } \quad s=j \omega
$$

Where, replacing the double vector $w d\left(\omega_{n}, \zeta_{\min }\right)$, the set of curves in the Bode diagram is obtained, which fulfill tracking specifications of the upper time limits and maximum overshoot, initially imposed.

6. Achieving the upper limit $T^{\prime}{ }_{U p p}(j \omega)$ in the frequency. On the Bode diagram above, the upper intersection (maximums in magnitude and in phase) of the different curves, defined by the double vector $w d\left(\omega_{n}, \zeta_{\text {min }}\right)$, is searched.

7. As the upper frequency limit curve $T^{\prime}{ }_{U p p}(j \omega)$ is made by the intersection of several $T(j \omega)$ curves, the way of describing its $T F$ is to approximate it using one of the following criteria, depending on the bandwidth and precision of interest :

a) Set the upper limit in the $W D$ with accuracy at low frequencies. That is, it is selected as $T_{U p p}(j \omega)$ the $T(j \omega)$ of maximum magnitude, at the lowest frequency in the frequency work band used. This approach will be named as restriction at low frequencies.

b) Set the upper limit in the $W D$ with accuracy at high frequencies. That is, it is selected as $T_{U p p}(j \omega)$ the $T(j \omega)$ of maximum magnitude, at the highest frequency of the frequency work band. This approach will be named as restriction at high frequencies.

c) Use rationalization of the frequency responses obtained in 6) to get an approach at the envelope of the upper limit $T_{U p p}(j \omega)$. It generates higher order $T F s$ using an order reduction method, such as defined in [1]. This approach will be named as restriction at the envelope approach.

8. Getting the final $T D$ parameters, from the $W D$ ones achieved, to match the original $T D$ requirements. That is, 


\section{Restriction at the Envelope Approach}

The envelope approach permits the reduction of higher order systems $T^{\prime}{ }_{U p p}(j \omega)$ to obtain the $T F$ simplified $T_{U p p}(j \omega)$. The envelope $T^{\prime}{ }_{U p p}(j \omega)$, representing the upper limit in frequency of the temporary tracking specifications imposed, may be rationalizing in the whole frequency work band, using an adequate method [11]. Firstly, the envelope must be expressed in complex form, within the frequency range: the reduced $T F$ solution obtained in a manually procedure with not too high order will be $T_{U p p}(j \omega)$.

Consider the relationship between the envelope input in complex form $T_{U p p}^{\prime}(j \omega)$ and its approach, the reduced transfer function $T_{U p p}(j \omega)$ :

$$
T_{U p p}(s)=\frac{z_{0}+z_{1} s+\ldots+z_{n} s^{n}}{p_{0}+p_{1} s+\ldots+p_{m} s^{m}} \approx T_{U p p}^{\prime}(s) \quad, \text { with } s=j w
$$

The different parameters in (18) are defined as,

- $T^{\prime}{ }_{U p p}$ : Complex input $T F$ with $[\mathrm{N}, 1]$ vector size.

- $\quad w$ : Frequency work band, with $[\mathrm{N}, 1]$ vector size.

- $\quad m$ and $n$ : Number of poles and zeros, respectively, for the reduced $T F T_{U p p}(s)$.

The equation (18) can be rearranged as:

$$
\left[p_{0}+p_{1} s+\ldots+p_{m-1} s^{m-1}\right]-\frac{1}{T_{U p p}^{\prime}}\left[z_{0}+z_{1} s+\ldots+z_{n} s^{n}\right]=-p_{m} s^{m}
$$

Using the $\mathrm{N}$ complex numbers in (19), one for each frequency $w$, from the input function $T^{\prime}{ }_{U p p}(s)$, we get $\mathrm{N}$ equations. Applying the rationalization process described by [7], we obtain values for the $m$ coefficients $p_{i}$ (poles) and $n$ coefficients $z_{i}$ (zeros).

Note that the number of poles $(m)$ and zeros $(n)$ are selected for the user.

At the end, we must use a polynomial evaluation over $T_{U p p}(s)$, to compare results in magnitude and phase with the original complex $T F T^{\prime}{ }_{U p p}(s)$ value. The differences are the errors in magnitude and phase of the process, for each frequency.

In Section 5, the practical development of this rationalization process can be downloaded. This program, RACWE.M in Matlab format, is described as follows:

$$
\text { function [numer, denom] }=\operatorname{racwe}\left(T^{\prime}{ }_{U p p}, w, n, m\right)
$$

From complex input $T^{\prime}{ }_{U p p}$, the function offers as output the transfer function $T_{U p p}$ (numer/denom), according to the supplied frequency vector $w$. The user can decide the order of the output $T F$, selecting the number of poles and zeros $m$ and $n$, it may contain. The order of the output $T F$ will influence in the difference between the output and the input in magnitude and phase, for each frequency. So, using different output orders and observing the differences obtained (errors), we can achieve reducing the order of the input TF with errors not too high and an adequate approach.

In this manual iteration process, we must consider the following conditions:

- The number of poles selected must be equal or greater than the number of zeros, to assure stability. 
- Also, to maintain stability there must be no RHP poles or zeros in the final output $T F$, nor poles with zero value. The program allows eliminating manually this type of poles/zeros and even too, those with insignificant values to reduce order.

Additionally, the program offers to the user the following possibilities:

- It permits to apply gain adjustment over the output $T F$ obtained.

- It presents numerical and graphical magnitude/phase differences between the input $T^{\prime}{ }^{\prime}{ }_{p p}(j \omega)$ and the output $T_{U p p}(j \omega)$. With this information, the user decides to stop the process or not.

- The process ends offering numerical and graphical temporary responses associated with $T_{U p p}(j \omega)$ obtained. With the visual information, the user decides if the approach is adequate or not.

\section{Application of the Technical for Tracking Specifications Translation of the Upper Frequency Limit}

Suppose tracking specifications given by the upper frequency limit, described by the set of temporal parameters $T D_{\text {original }}\left(y_{\max }=2.3, r_{\max }=1.8\right.$, dev $= \pm 1.8 \%$ ), ie, an input step of amplitude 1.8 must generate a step response with a maximum amplitude value 2.3 (maximum overshoot equivalent of $27.78 \%$ ), with maximum rise and settlement times, by default of $5 \mathrm{~s}$ and $20 \mathrm{~s}$, respectively, and the admissible tolerance of $\pm 1.8 \%$. Applying the technique described above, with the downloadable programs in Matlab as indicated in Section5, it is obtained:

- $2^{\text {nd }}$ order responses $T(j \omega)$, from $w d\left(\omega_{n}, \zeta_{\min }\right)$, which are fitted to the $T D_{\text {original }}$ parameters, given by the upper time limit; these are described, in this case, with a total of 39 TFs.

- Bode diagram of the $2^{\text {nd }}$ order responses $T(j \omega)$, from the double vector $w d\left(\omega_{n}, \zeta_{\text {min }}\right)$. Results in Figure 2.

- Sets of $T D$ responses, equivalent to those $T(j \omega)$ systems in the WD. Results are presented in Figure 3.
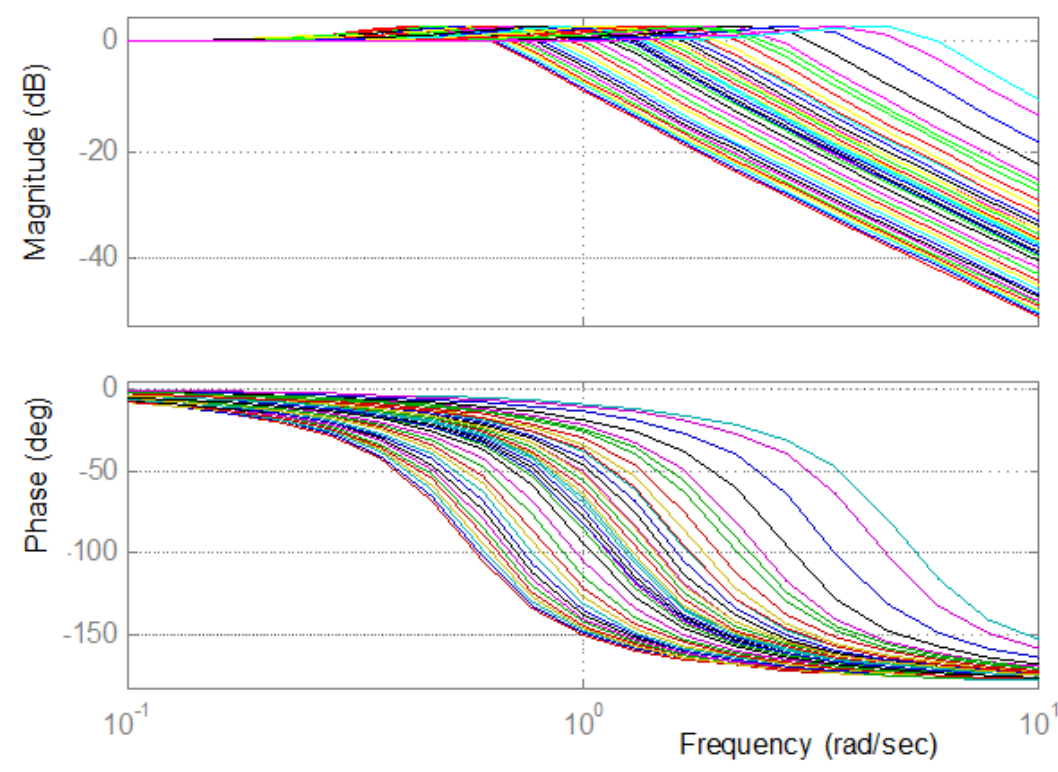

Figure 2. $2^{\text {nd }}$ Order Responses $\mathrm{T}(\mathrm{j} \omega)$ 
- Upper limit $T^{\prime}{ }_{U p p}(j \omega)$ in the frequency domain. It is obtained looking for maximums in magnitude and phases, throughout the frequency work band, over the 39 TFs achieved. Results in Figure 4.

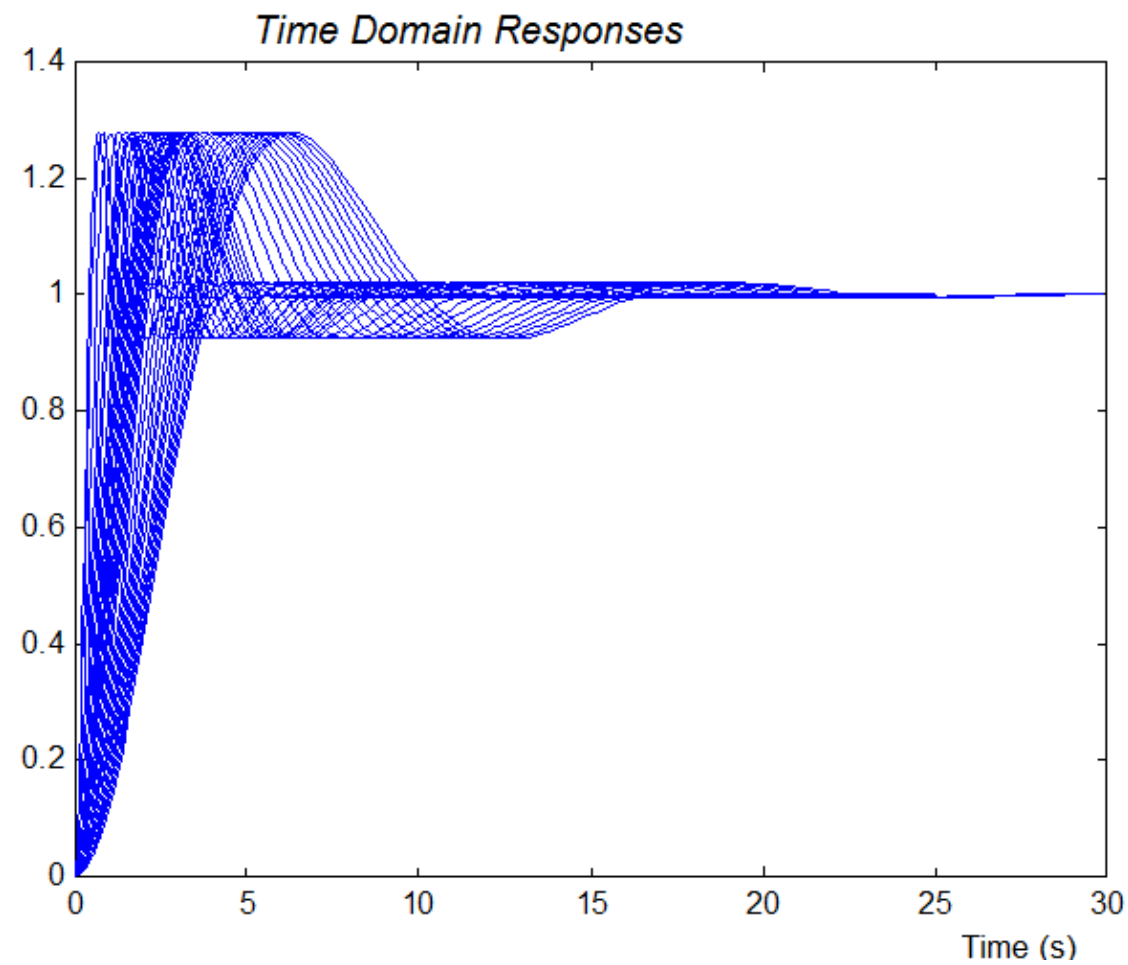

Figure 3. Set of Temporary Responses, Equivalent to the Systems T(jw)

- Upper limit $T_{U p p}(j \omega)$ in the frequency domain, considering restriction at low frequencies, ie, from the curves of maximum magnitude in the low frequency area. Results in Figure 5.

- Obtaining curve and final TD parameters that fit to the original TD, applying restriction at low frequencies. Temporary results in Figure 6.

- Upper frequency limit defined as the most restrictive curve $T(j w)$, at low frequencies,

$$
\left(\frac{\text { Num }_{\text {max }}}{\text { Den }_{\max }}\right)_{L o}=\frac{0.2915}{s^{2}+0.4077 s+0.2915}
$$




\section{Maximum magnitudes/phases}

$\mathrm{Gm}=21.199 \mathrm{~dB}$ (at $16.77 \mathrm{rad} / \mathrm{sec}$ ), $\mathrm{Pm}=64.556 \mathrm{deg}$. (at $5.9963 \mathrm{rad} / \mathrm{sec}$ )
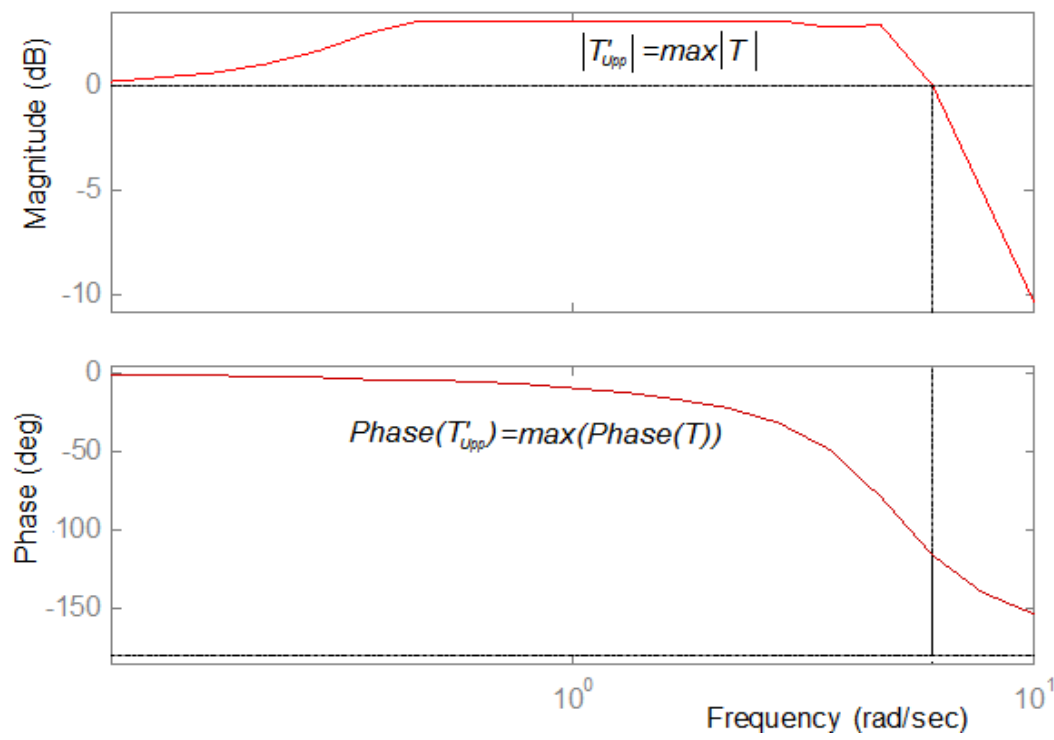

Figure 4. Upper Limit T'upp(jw) in the WD
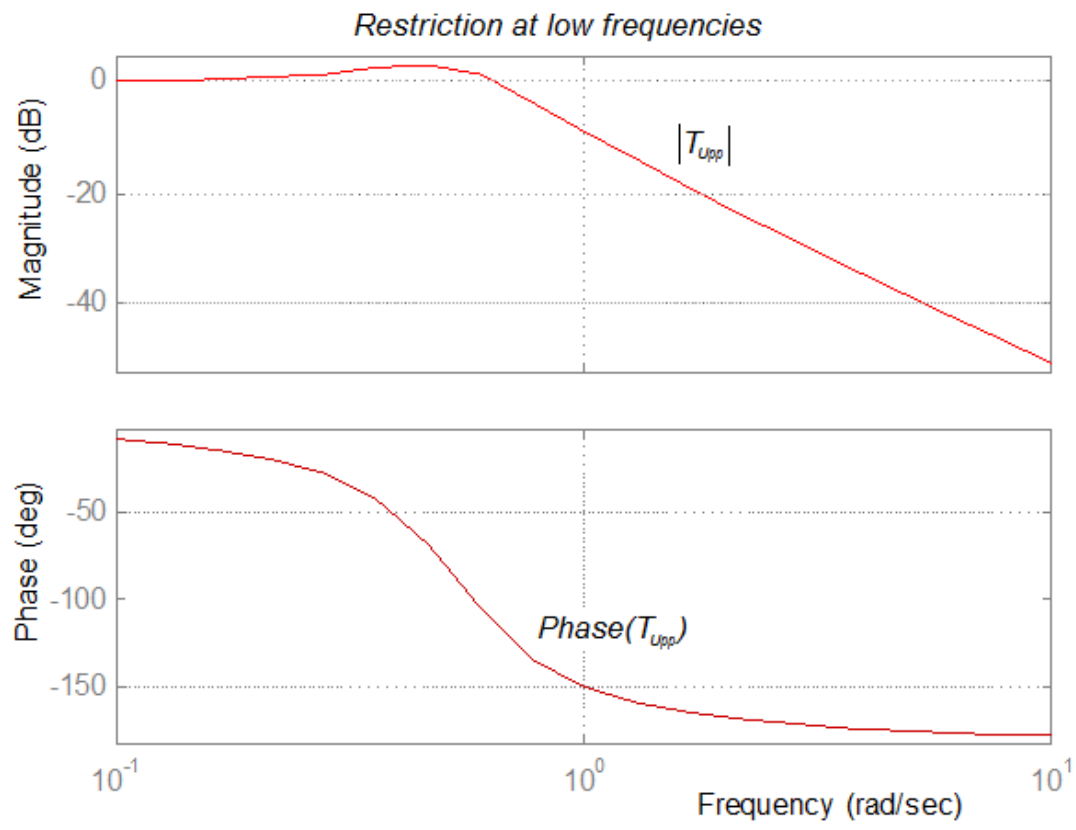

Figure 5. Upper Limit $T_{u p p}(j \omega)$ in the WD, Considering Restriction at Low Frequencies 


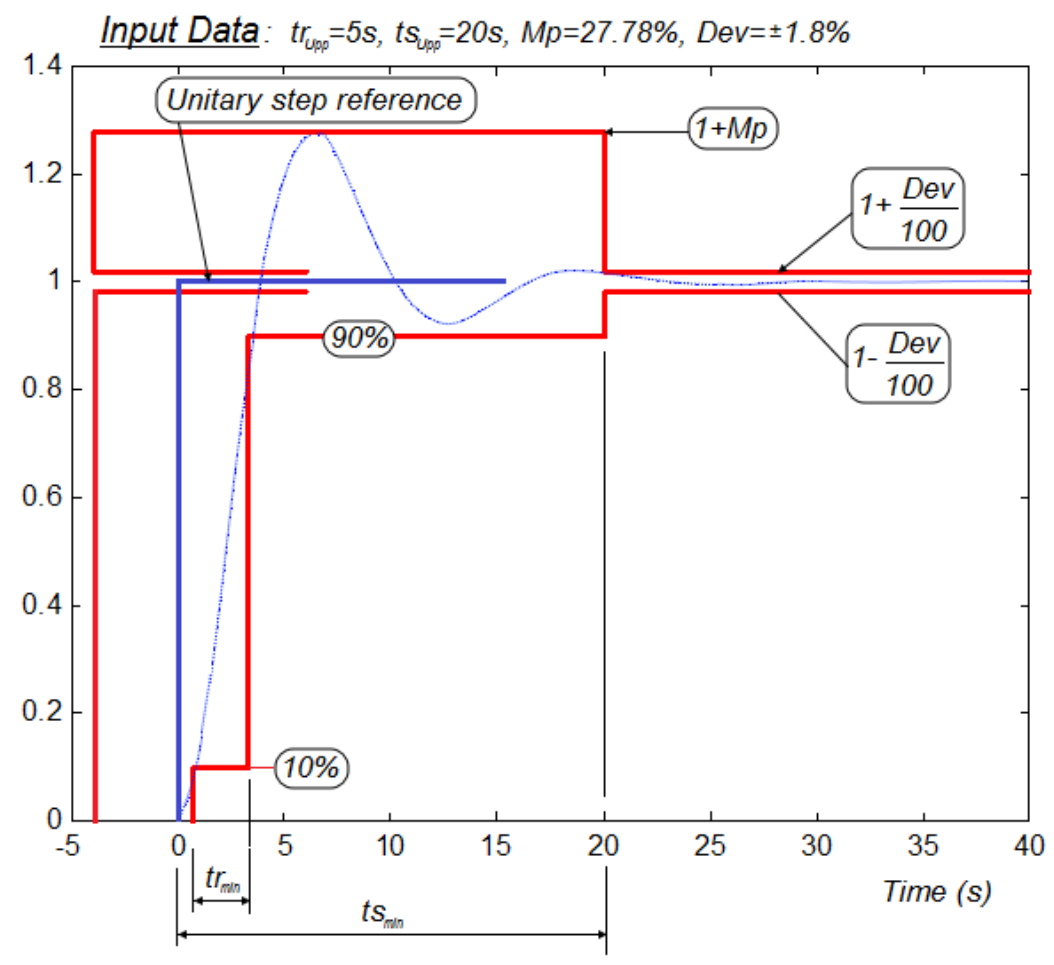

Response : $\operatorname{tr}_{\min }=2.6746 \mathrm{~s}, t s_{\min }=20 \mathrm{~s}, M p_{\mathrm{mas}}=27.78 \%$

Figure 6. Final Temporary Response Associated with $\mathrm{T}_{\text {upp }}(\mathrm{J} \omega)$, Considering Restriction at Low Frequencies

- Upper limit $T_{U p p}(j \omega)$ in the frequency domain, considering restriction at high frequencies, ie, from the curves of maximum magnitude in the high frequency area. Results in Figure 7.
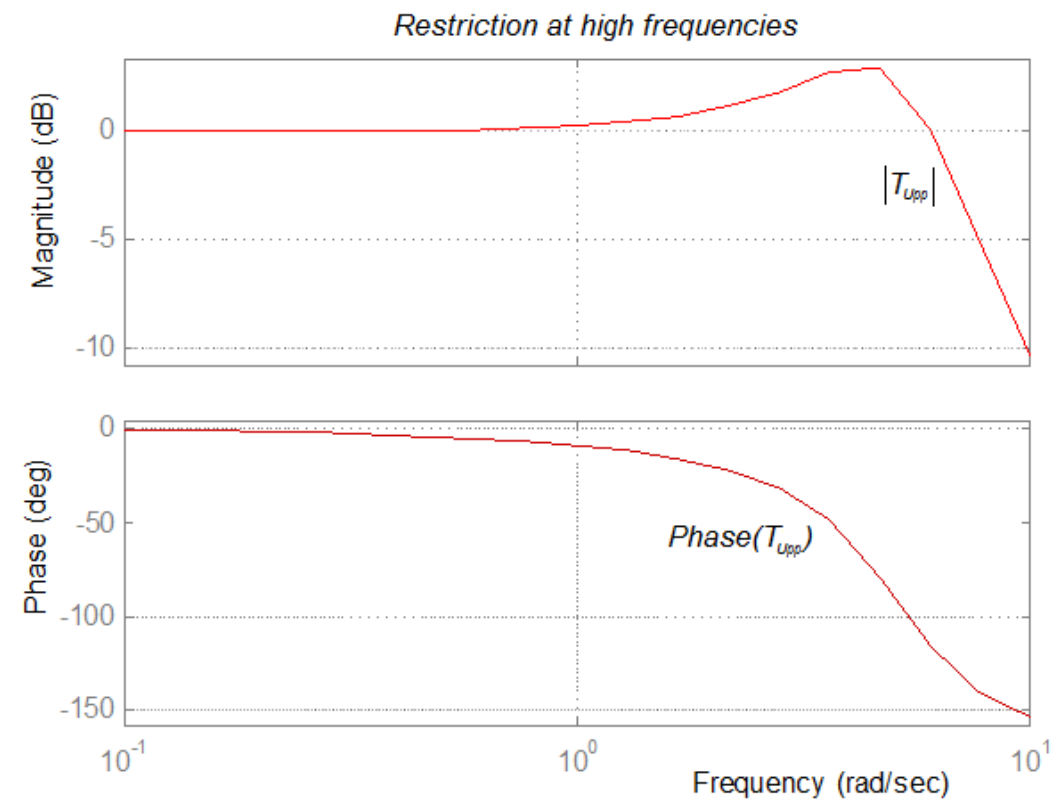

Figure 7. Upper Limit $T_{U p p}(j \omega)$ in the WD, Considering Restriction at High Frequencies 
- Curve and final TD parameters that fit to the original TD, applying restriction at high frequencies. Temporary results in Figure 8.

- Upper frequency limit defined as the most restrictive curve $T(j w)$, at high frequencies,

$$
\left(\frac{N u m_{\text {max }}}{\text { Den }_{\text {max }}}\right)_{H i}=\frac{25.15}{s^{2}+3.787 s+25.15}
$$

- Upper limit $T_{U p p}(j \omega)$ in the frequency domain, considering restriction at the envelope approach, ie, rationalizing the real limits until $T F$ has not got too high order. Results in Figure 9. Graphical magnitude/phase differences between $T^{\prime}{ }_{U p p}(j \omega)$ and $T_{U p p}(j \omega)$ are expressed in Figure 10. We are obtaining a maximum magnitude error of $0.3342 \mathrm{rad}$ and a maximum phase error of 8.38 degrees.

- Curve and final TD parameters that fit to the original TD, applying restriction at the envelope approach. Temporary results in Figure 11.

- Upper frequency limit obtained rationalizing the upper intersection in the set $T(j \omega)$, defined with two poles and one zero. There are other possible stable $T F$, but this one offers the closest maximum overshoot $\left(M p_{\text {final }}=25.41 \%\right)$ respect the required, with minimum approach errors and not too high order.

$$
\left(\frac{N u m_{\max }}{D e n_{\max }}\right)_{E n v}=\frac{0.3099 s+21.35}{s^{2}+3.697 s+21.35}
$$

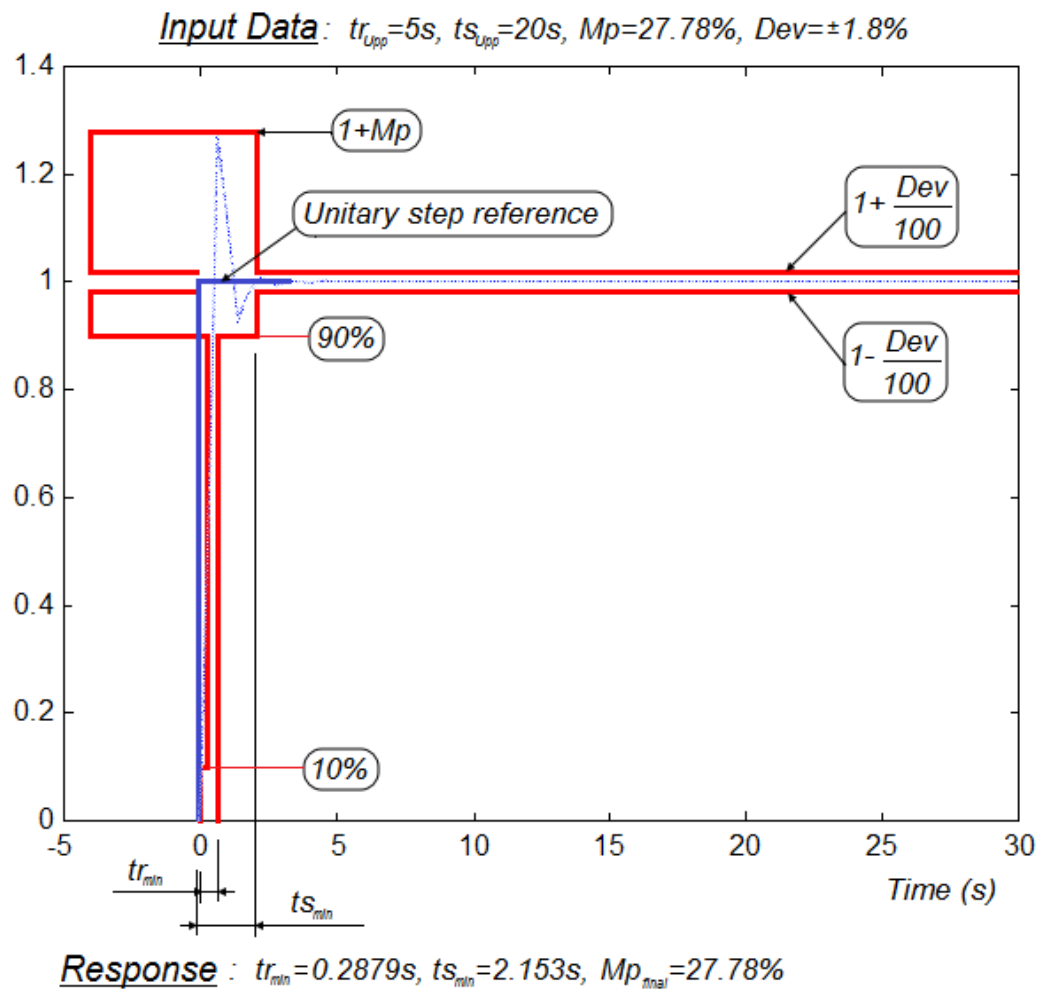

Figure 8. Final Temporary Response Associated with $\mathrm{T}_{\mathrm{upp}}(\mathrm{j} \omega)$, Considering Restriction at High Frequencies 

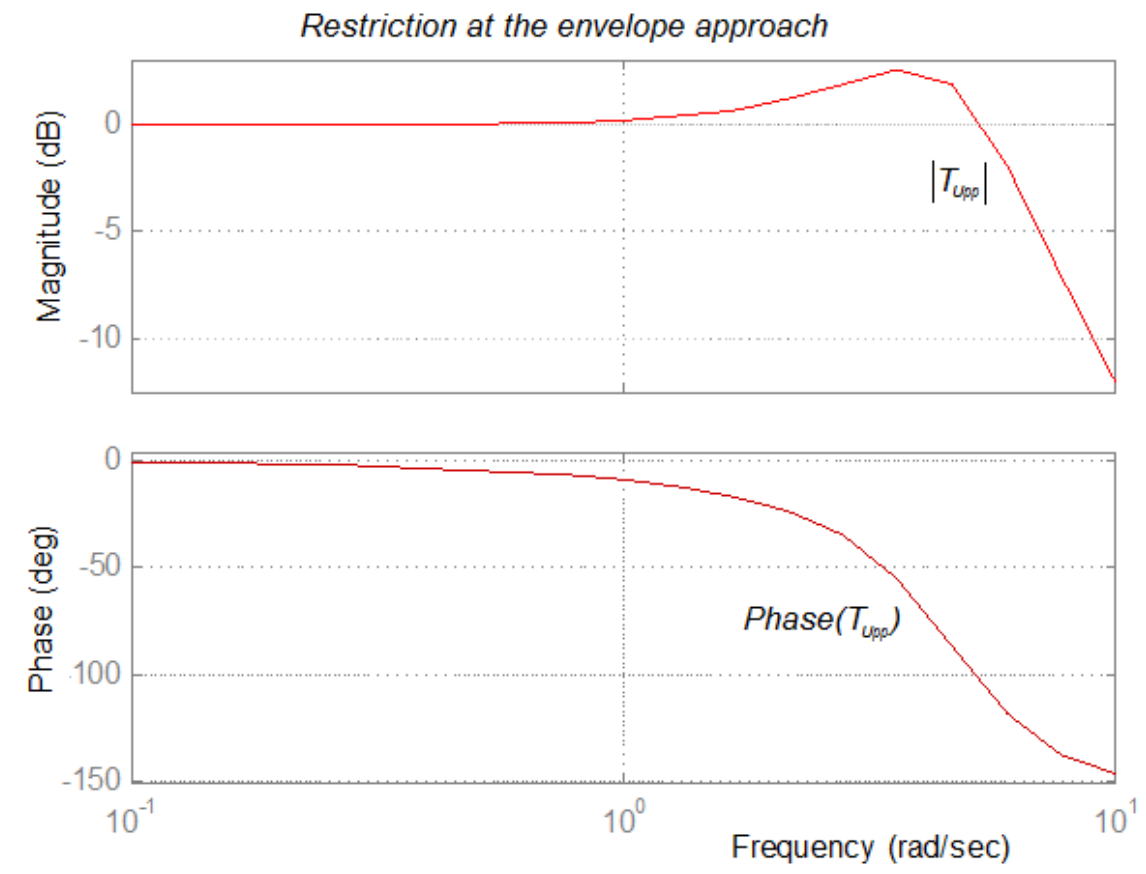

Figure 9. Upper Limit $T_{U p p}(j \omega)$ in the WD, Considering Restriction at the Envelope Approach

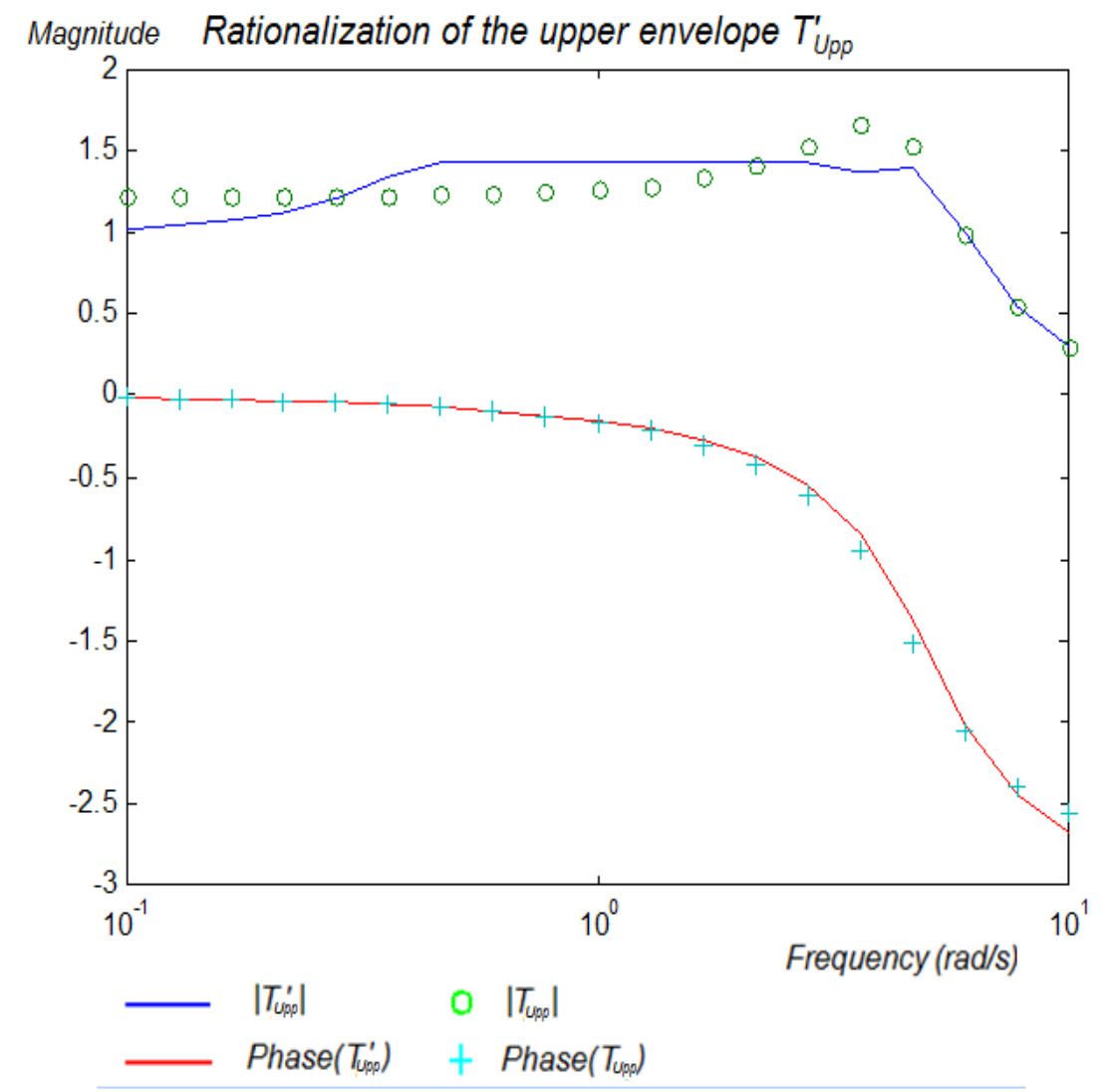

Figure 10. Graphical differences in Magnitude and Phase between T'upp(jw) and the Upper Envelope Approach TF Tupp $(j \omega)$ 


\section{Programs Development}

The necessary programs to verify the above example and any other, for tracking temporary specifications translation to frequency domain, have been developed in Matlab format.

"Temporary Tracking Specifications Conversion to Obtain the Upper Frequency Limit" can be downloaded from the next URL:

https://www.dropbox.com/sh/16w7ujosn9yet7v/AADmlrplf1489cK7Uaa6ODvca?dl=0

The application starts with the program STEPTRAC.M

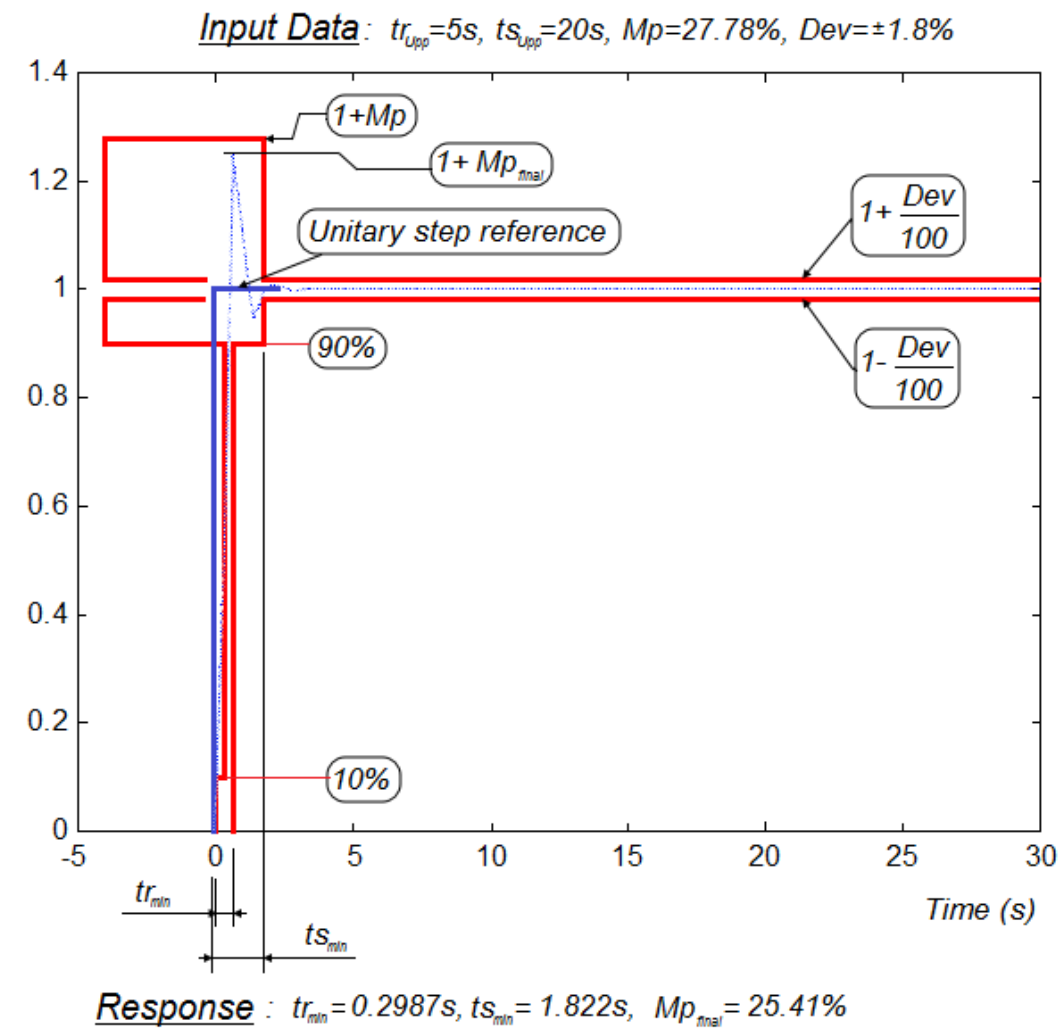

Figure 11. Final Temporary Response Associated with $T_{u p p}(j \omega)$, Considering Restriction at the Envelope Approach

\section{Conclusions}

Techniques for transferring rigorous specifications from $T D$ to $W D$ are more important than traditionally they have been considered: control methodologies with temporary requirements, but whose design stage is performed in the frequency domain, supplement the consequences of shortcomings in this field using iterative processes, long and complex sometimes, which still affect the final result as overdesign and relaxation of specifications, that cannot be fulfilled.

These conversion techniques between domains will allow having to work with less iteration until a suitable solution is achieved, if it exists, since the controller searched in the $W D$ can match to more exactly frequency conditions from the beginning. Furthermore, being the deviation of the requirements demanded in the design stage minimum, the overdesign cost is reduced.

It has been developed here an automatic specific procedure for precision conversion of tracking specifications from time to frequency, to get the upper frequency limit using 
approach to a $2^{\text {nd }}$ order system with subcritical damping. On the other hand, it can be chosen the translation option with manually approach to a higher order system too.

The technique generates the $T F$ associated to an upper frequency limit that, with a step input of value $r_{\max }$, produces a subcritical damped response with maximum amplitude $y_{\max }$, so that, the overshoot $M p$ will be a particular one. Also it uses, as additional input parameters, maximum reference times of rise and settlement, $t r_{u p p}$ and $t s_{u p p}$, respectively. From these times the search begins by inverse interpolation of the response frequency associated to the more appropriate upper limit. The process and final result depend on the selected approach, which determines the area of the bandwidth where maximum accuracy is required: low or high frequency (specific area in the bandwidth) or envelope (whole bandwidth).

The lower frequency limit required by some type of tracking specifications is often described in temporary terms of overshoot response to a given step input. Considering the principles of the technique presented in this paper, it can be developed a specific method to get this kind of tracking specification in the $W D$.

The methodology described basing on temporary parameters inverse interpolation, can be simplified applying classical hypothesis, although this implies reducing accuracy.

Sensitivity specifications described in time are characterized by a disturbance input which should produce a damped impulse response, with very specific amplitude and fading characteristics. The methodology for temporary specifications conversion to frequency using inverse interpolation can be applied in this case too, by timely adaptation.

\section{References}

[1] J. Aranda and J. Joglar, "Application of QFT order reduction methodology in the control of an autonomous marine vehicle", Journal of Maritime Research, vol. 11, no. 3, (2014), pp. 67-78

[2] J. F. Bonnans, J. C. Gilbert, C. Lemaréchal and C. A. Sagastizábal, "Numerical optimization: Theoretical and practical aspects", Universitext, Berlin: Springer-Verlag., (2006), pp. xiv+490. DOI: 10.1007/ 978-3-540-35447-5. ISBN 3-540-35445-X. MR 2265882.

[3] C. Borghesani, Y. Chait and O. Yaniv, "The QFT control desing Toolbox - for use with MATLAB", Terasoft Inc. (2003).

[4] J. D'Azzo and C. H. Houpis, "Linear control system analysis and design conventional and modern", Cap18, $4^{a}$ Ed, McGraw-Hill, New-York, (1995).

[5] M. A. Franchek and P. A. Herman, "Direct connection between time-domain performance and frequency-domain characteristics", International Journal of Robust and Nonlinear Control, vol. 8, (1998), pp. 1021-1042.

[6] M. Gil-Martínez, "Síntesis de controladores robustos mediante el análisis de la compatibilidad de especificaciones e incertidumbre", Ph.D. thesis, Universidad de la Rioja, Spain, (2008).

[7] I. M Horowitz, "The Quantitative Feedback Theory”, QFT Publishers, Boulder, Colorado, vol. 1, (1992)

[8] I. M. Horowitz and M. Sidi, "Synthesis of feedback systems with large plant ignorance for prescribed time domain tolerances", International Journal of Control, vol. 16, (1972), pp. 287-309.

[9] C. H. Houpis and S. J. Rasmussen, "Quantitative Feedback Theory: fundamentals and applications", Marcel Dekker, New York, (1999).

[10] J. Joglar-Alcubilla, "Contribución al estudio de diseño de sistemas de control mediante QFT: aplicaciones al diseño de sistemas de control de vuelo y navegación”, Ph.D, thesis, ETSI Informática, UNED, Spain, (2015).

[11] J. Joglar-Alcubilla, "Generic tracking specifications translation from time domain to frequency domain", Arxiv: 1601.00346 [cs.SY], (2016)

[12] K. R. Krishnan and A. Cruickshanks, "Frequency-domain design of feedback systems for specified insensitivity of time-domain response to parameter variation", International Journal of Control, 25(4), (1977), pp. 609-620.

[13] O. D. I. Nwokah and C. H. Yau, "Quantitative Feedback Design of Decentralised Control Systems", J. Dyn Syst-T ASME, vol. 115, (1993), pp. 452-464.

[14] K. Ogata, "Ingeniería de control moderna", Prentice Hall, (1993).

[15] M. Sobhani and S. Jayasuriya, "Incorporating Right Half-Plane Poles and Zeros in a Frequency Domain Design Technique", Journal of Dyn Syst-T ASME, vol. 116, no. 4, (1994), pp. 593-601.

[16] S. Yi S., P. W. Nelson and A. Galip Ulsoy, "Robust Control and Time-Domain Specifications for Systems of Delay Differential Equations via Eigenvalue Assignment", Journal of Dyn Syst-T ASME, 132(3), (2010), DOI:10.1115/1.4001339. 
[17] Y. Zhao and S. Jayasuriya, "A New Formulation of Multiple-Input Multiple-Output Quantitative Feedback Theory", J. Dyn Syst-T ASME, vol. 118, (1996), pp. 748-752.

\section{Author}

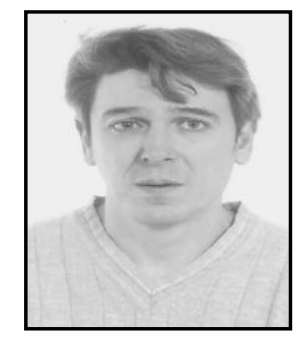

Javier Joglar-Alcubilla, works as a professor in the avionics department of the Barajas Institute in Madrid, Spain. He received his B.Sc in Aeronautics Engineering from Polytechnic University at Madrid, Spain, in 1990 and his M. Sc. degree in Electronics and Automation Physics from UNED, Spain, in 1994. He received his $\mathrm{Ph} . \mathrm{D}$. degree in Informatics Engineering from ETSII at UNED, Spain, in 2015. He is the author of nine technical books. 
International Journal of Control and Automation Vol.10, No.4 (2017) 